\title{
Gewerkschaften in Nigeria seit 1977 - Probleme und Perspektiven
}

von Christoph Muermann

Nigeria ist für die BRD mehr als ein exotischer Staat nahe am Äquator. Das Land, in dem mit 80 Mio Einwohnern ein Fünftel aller Afrikaner leben, ist zu einem unserer wichtigsten Erdöllieferanten geworden.

Deutsche Unternehmen haben zunehmend den nigerianischen Markt entdeckt. So beschäftigen allein VW oder Mercedes-Benz in ihren Zweigstellen in Nigeria Tausende von einheimischen Arbeitskräften.

Wissen um die Probleme und Perspektiven der Lohnabhängigen und ihrer Gewerkschaften in Nigeria scheint daher in einer Zeit der Internationalisierung der Produktion immer wichtiger zu werden.

Der 'Nigerian Labour Congress ${ }^{1}$ mit seinen 3,5 Mio Mitgliedern (von insgesamt 5,5 Mio Lohnabhängigen) ist schon heute eine der mitgliederstärksten afrikanischen Gewerkschaften ${ }^{2}$ und somit ein wichtiger Bestandteil weltweiter gewerkschaftlicher Interessenvertretung.

\section{Rahmenbedingungen gewerkschaf tlichen Handelns}

Obwohl die nigerianischen Gewerkschaften auf eine traditionsreiche Geschichte zurückblicken können - so fand der erste Streik schon 1897 in Legos statt - gewinnen sie zahlenmäßig erst mit der beginnenden Industrialisierung Anfang der 60er Jahre an Bedeutung. ${ }^{3}$ Da die Masse der Bevölkerung noch von der Subsistenzlandwirtschaft lebte (und auch heute noch lebt) und die wenigen Lohnabhängigen zumeist zugleich Staatsangestellte waren, konzentrierte sich die gewerkschaftliche Organisation auf den staatlichen Sektor. Mit der kapitalintensiven Industrialisierung, die zu Beginn der 60er Jahre einsetzt, nimmt auch die Zahl der Lohnabhängigen im privaten Sektor zu. Diese Entwicklung verstärkt sich dann mit dem 'Ausbruch des Olboomsı. Waren es in den 60er Jahren v. a. Multinationale Konzerne, die in Nigeria investierten, treten nun als Investoren zu-

1 Der Nigerian Labour Congress (NLC) läßt sich von der Organisationsstruktur in etwa mit dem DGB vergleichen.

2 Zahlenangaben nach Dr. Osunde, Deputy General Secretary des NLC, in einem Interview mit dem Verfasser am 25. 9. 80. Es ist allerdings festzuhalten, daß viele Statistiken und Zahlenangaben in Nigeria - gerade im gewerkschaftlichen Bereich - auf Schätzungen beruhen.

3 Als Einführung in die Geschichte der Gewerkschaften Nigerias seien zwei Werke genannt: "Labour and Politics in Nigeria 1945-71", Robin Cohen, London, Heinemann, 1974 und "The Trade Union Movement in Nigeria", Wogu Ananaba, London, Hurst \& Co, 1969. 
nehmend halbstaatliche Institutionen und daneben private nigerianische Unternehmen auf. ${ }^{4}$

Die wichtigsten Sektoren der industriellen Produktion sind heute die Nahrungsmittelund Textilindustrie, die Baubranche und die Metallwarenindustrie. Obwohl der moderne industrielle Sektor in den 70er Jahren Rekordproduktionszuwächse zu verzeichnen hat, beträgt sein Anteil am BIP 1980 doch nur $8 \% .{ }^{5}$ Auf Grund des kapitalintensiven Investitionsmusters sind dort zudem nur relativ wenig Arbeitskräfte beschäftigt.

In den industriellen Zentren der Städte absorbiert der sog. traditionelle Sektor ${ }^{6}$ damit nach wie vor die meisten Arbeitskräfte. Er weitet sich in den 70er Jahren sogar besonders stark aus.

(1) Zur wirtschaftlichen Entwicklung Nigerias vgl. im einzelnen etwa: Gavin Williams, "Nigeria, a Political Economy", London, 1976; R. O. Ekundare, "An Economic History of Nigeria 1860-1960, London, 1973; Ludwig Schätzl, "Industrialization in Nigeria, A spatial analysis", München 1973.

Gegenüber diesen im traditionellen und modernen (staatlichen und privaten) Sektor Beschäftigten, ist aber festzuhalten, daß die überwiegende Mehrzahl der Erwerbstätigen weiterhin von der Landwirtschaft lebt.

Die Strukturen dieser Entwicklung bestimmen in vielerlei Hinsicht die Rahmenbedingungen gewerkschaftlichen Handelns in Nigeria.

Der Staat und seine Institutionen dominieren nach wie vor das wirtschaftliche Geschehen. Er ist Hauptbeschäftigungsgeber. Nimmt man die halbstaatlichen Unternehmen hinzu, sind etwa $60 \%$ aller Beschäftigten bei ihm angestellt. ${ }^{7}$ Hieraus ergibt sich auch die of tominierende Rolle der Gewerkschaften des staatlichen Sektors. Bei Tarifauseinandersetzungen setzten sie bisher Lohnerhöhungen oft zuerst durch, denen dann die Gewerkschaften im privaten modernen Sektor folgten. Die Lohnabhängigen im traditionellen Sektor mit seinen vielen Kleinstbetrieben sind dagegen kaum gewerkschaftlich organisiert; ihre Löhne liegen weit unter den sonstigen Mindestlöhnen.

Der industrielle Boom hat die Landflucht v. a. in Zentren wie Lagos, Ibadan, Kaduna und Port Harcourt beschleunigt. Eine Verslummung von bisher nicht gekanntem Ausmaß und ein rapides Anwachsen der Arbeitslosenzahl war die Folge. Insbesondere die Vielzahl der Beschäftigungslosen stellt eine große Hypothek für jede aktive Tarifpolitik der Gewerkschaften dar.

Andererseits hat der industrielle Boom aber auch zu einem Mangel an qualifizierten Ar-

Zur wirtschaftlichen Entwicklung Nigerias vgl. im einzelnen etwa: Gavin Williams, "Nigeria, a Political Economy", London, 1976; R. O. Ekundare, "An Economic History of Nigeria 1860-1960, London, 1973; Ludwig Schätzl, "Industrialization in Nigeria, A spatial analysis", München 1973.

5 Guidelines For The Fourth National Development Plan, Lagos 1980, S. 17.

6 Unter dem "traditionellen" Sektor sollen, ausgehend davon, daß in den nigerianischen Städten eine Vielzahl von Klein- und Kleinstbetrieben existieren, die zwar arbeitsintensiv aber mit.nur wenigen Beschäftigten produzieren, und in Anlehnung an die in Nigeria übliche Klassifizierung diejenigen Betriebe mit weniger als 10 Beschäftigten verstanden werden.

7 Angabe von Dr. Osunde geg. dem Verfasser, 23. 9. 80. 
beitskräften geführt. ${ }^{8}$ Trotz dieses generellen Booms produzieren aber einige industrielle Branchen, so v. a. die Textilindustrie im internationalen Vergleich zu teuer. So wurden allein 1980 in den großen Textilfabriken des Nordens Tausende von Arbeitern entlassen. Andere Probleme ergeben sich für die Gewerkschaften aus der Existenz der halben Mio Gastarbeiter, die vor dem noch größeren Elend in den westafrikanischen Nachbarstaaten nach Nigeria geflohen sind und nun oft als Lohndrücker eingesetzt werden.

\section{Neue gewerkschaftliche Strukturen}

Gegenüber diesen wirtschaftspolitischen Bestimmungsfaktoren, haben sich aber die organisatorischen und finanziellen Ausgangsbedingungen für die gewerkschaftliche Arbeit in Nigeria seit 1977/78 entscheidend verbessert. Noch in der Zeit der Militärregierung (die im Oktober 1979 von einer Zivilregierung abgelöst wurde) ist ein einheitlicher Dachverband (NLC) gegründet worden, dem 42 Einzelgewerkschaften, die auf Industriebasis organisiert sind, angeschlossen sind. Vorher gab es allein auf nationaler Ebene 4 rivalisierende Dachverbände, von denen die beiden größten, ULC und NTUC, nicht nur den internationalen Verbänden IBFG und WGB angeschlossen waren, sondern oft auch auf deren finanzielle Unterstützung angewiesen waren. ${ }^{9}$ Die Zahl der ihnen angeschlossenen Einzelgewerkschaften belief sich vor der Neuorganisation sogar auf über Tausend. Der Einflußbereich dieser vielen Minigewerkschaften reichte aber oft nicht über einen Betrieb hinaus. Entsprechend schwach war dann die Verhandlungsposition dieser Hausgewerkschaften gegenüber dem jeweiligen Arbeitgeber.

Der Neugründung und organisatorischen Straffung der nigerianischen Gewerkschaften, die die Militärregierung durch zwei Dekrete ${ }^{10} 1977 / 78$ einleitete, waren gewerkschaftliche Bemühungen in die gleiche Richtung vorausgegangen; schon 1974 hatten sich Vertreter der vier Dachverbände in einer gemeinsamen Deklaration (Apena Cemetry Declaration) für einen gemeinsamen Neuanfang ausgesprochen. Ein erster Versuch in dieser Richtung wird aber von den Militärs wegen angeblicher Korruption beteiligter Gewerkschafter 1976 zunichte gemacht. Erst am 28. Februar 1978 kommt es endgültig zur Gründung des NLC. Hassan Sunmonu wird zu seinem ersten Präsidenten gewählt. Zugleich werden die 42 Industriegewerkschaften und 15 Assoziationen für höhere Angestellte gegründet. Obwohl die Militärregierung nach Einschätzung nigerianischer Gewerkschafter mit dieser Neugründung wohl eher einen Ansprechpartner bzw. ein Disziplinierungsinstrument in die Hand bekommen wollte, hat sich der NLC nach einer Anlaufphase als eigenständige Kraft entwickelt und stellt zunehmend eine ernstzunehmende politische Kraft dar.

8 Vgl. Guidelines For The Fourth National Development Plan, Lagos 1980, S. 82 f.; West Africa vom 16. 3. 81, S. $543 \mathrm{f}$.

9 Vgl. den instruktiven "Report of the Tribunal of Inquiry into the Activities of Trade Unions" (Adebiyi-Report), Lagos 1977.

10 Decree No. 44 von 1976 und Decree No. 22 von 1978, in Kraft gesetzt am 3. 8. 77. 
Organisatorisch ist der NLC im Moment noch mit dem Aufbau seiner Unterabteilungen beschäftigt, der erst nach dem Umzug in größere Räumlichkeiten im Frühjahr 1981 voll abgeschlossen sein wird. Zur Verwirklichung vieler Projekte, wie dem Aufbau von Kredit- und Konsumgenossenschaften, der Gründung einer eigenen Bank und der Forcierung der gewerkschaftlichen Bildungsarbeit fehlen oft noch die finanziellen Mittel.

Die Regierung, die 1978 zunächst mit 1 Mio Naira ${ }^{11}$ die Entstehung der Gewerkschaften unterstützt hatte, subventioniert den NLC heute nur noch in geringem Umfang; so z. B. durch die Erstellung eines neuen Gebäudes für die Zentralverwaltung. Den Großteil seiner Einnahmen bezieht der NLC von den 42 angeschlossenen Einzelgewerkschaften, die meist $10 \%$ ihres Budgets an diesen weiterleiten. Da auch das höchste Gremium des NLC, der National Executive Council, mehrheitlich von den Mitgliedern der Einzelgewerkschaften besetzt wird ${ }^{12}$ ist gewährleistet, daß NLC und Einzelgewerkschaften stark ineinander integriert sind.

\section{Die Einzelgewerkschaften}

Die 42 Einzelgewerkschaften sind oft von recht unterschiedlicher Größe. Mammutgewerkschaften des staatlichen Sektors stehen Minigewerkschaften wie die "Gewerkschaft der Pensionäre" gegenüber, deren Entstehung oft nicht nach funktionalen Gesichtspunkten, sondern nur historisch zu erklären ist. So gibt es allein im öffentlichen Bereich drei Gewerkschaften, was mitunter zu Abgrenzungsschwierigkeiten führt. Viele der größten Gewerkschaften bestehen im staatlichen Bereich, wie die:

- Nigeria Civil Service Union 250000 Mitglieder

- Nigeria Union of Civil Service, Typists and Allied Staff

- Nigeria Union of Teachers 112000 Mitglieder

- Civil Service Technical Workers' Union of Nigeria 60000 Mitglieder

- Nigeria Union of Railwaymen

- Nigeria Union of Local Government Employees

72000 Mitglieder 30000 Mitglieder nicht bekannt

Im privaten Sektor sind bedeutende Gewerkschaften die:

- Nigeria Union of Constructive \& Civil Engineering Workers $210000(\max )$ Mitglieder

- National Union of Textile, Garment and Tailoring Workers

62000 Mitglieder

- National Union of Food, Beverages and Tobacco Employees nicht bekannt

11 Naira entsprach 1980 etwa 3,20 DM. Die reale Kaufkraft liegt - gemessen am inoffiziellen Kurs - etwa niedriger.

12 Vgl. im einzelnen: Nigeria Labour Congress: Constitution, S. 6 f. und 29 f. 
- National Union of Shop and

Distributive Employees

nicht bekannt

- Footwear, Leather and Rubber

Products Workers' Union

30000 Mitglieder ${ }^{13}$

Das Mitgliederpotential ist aber bei den meisten Gewerkschaften noch weitaus größer und nimmt rasch zu. So schätzt etwa die Textilgewerkschaft die Zahl der in größeren Textilbetrieben beschäftigten Arbeiter auf über $120000 .{ }^{14}$ Die Schwierigkeit der Rekrutierung liegen für die Gewerkschaften dabei v. a. im privaten Sektor und hier wieder bei den kleineren Betrieben. Nach Einschätzung nigerianischer Gewerkschafter läßt sich zudem ein "Gefälle" in der Haltung der Arbeitgeber gegenüber den Gewerkschaften feststellen, je nachdem ob der Betrieb von europ./amerikanischem Management, libanesisch/chinesischen Unternehmern oder nigerianischen Einheimischen geleitet wird. Das europäische Management ist an einem geordneten Produktionsablauf in den meist größeren Betrieben interessiert, duldet die Gewerkschaften daher in der Regel und versucht sie als Disziplinierungsinstrument zu benutzen. Die von einheimischen Nigerianern ("long-legs") geleiteten Firmen, versuchen dagegen das Entstehen von Gewerkschaften von vorneherein $\mathrm{zu}$ verhindern und werden daher oft als "not-unionizable ${ }^{15}$ eingeschätzt. Diese Unterschiede in der Strategie hindern die europäischen Firmen, die of $t$ mit Mißtrauen gegenüber ihrem Engagement in Nigeria rechnen müssen, allerdings nicht daran als Personalmanager meist nur Nigerianer einzustellen, da diese mit den Gewerkschaften und Lohnabhängigen "nigerianisch" umgehen können. Auch haben große deutsche Firmen, wie etwa Julius Berger die Anerkennung ihrer Gewerkschaften oft bis 1978 hinausgezögert, als mit der Neustrukturierung der Gewerkschaften eine Anerkennung in praxi nur noch schwer zu verhindern war.

\section{Organisation und Finanzen der Einzelgewerkschaften}

Die angesprochene Neustrukturierung der Gewerkschaften 1977/78 brachte für die 42 Einzelgewerkschaften einschneidende Veränderungen und Verbesserungen mit sich. Jede von ihnen ist jetzt zum ersten Mal für einen mehr oder weniger klar umgrenzten Bereich im privaten oder staatlichen Sektor ausschließlich zuständig. Es ist gesetzlich verankert, daß die Mitgliedsbeiträge in den Betrieben mit gewerkschaftlicher Organisation automatisch vom Arbeitgeber vom Lohn abgezogen werden und an die entsprechenden Einzelgewerkschaften überwiesen werden. Als gewerkschaftlich organisiert werden i. d. R. die Betriebe angesehen, in der die Mehrheit der Arbeitnehmer sich dafür ausgesprochen

13 Die Zahlen beruhen auf eigenen Erhebungen, die wiederum of t nur Schätzungen der Einzelgewerkschaften wiedergeben. Da sowohl der Einfluß der Einzelgewerkschaften auf den NLC als auch die von ihnen zu leistenden Zahlungen an den NLC sich nach der Mitgliederzahl richten, ergeben sich mitunter differierende Angaben.

14 Angabe von Mallam Bello Mohamed, Präsident der Textilarbeitergewerkschaft.

15 Also als nicht gewerkschaftlich organisierbar. 
hat. Jeder Betriebsangehörige hat allerdings das Recht aus der Gewerkschaft auszutreten. ${ }^{16}$ Mitgliedsbeitrag ist bei fast allen Gewerkschaften momentan 1 Naira pro Monat, was etwa 1/100 des Lohnes der meisten Gewerkschafter entspricht. ${ }^{17}$

Dieses staatlich garantierte Lohnabzugsverfahren scheint nach einigen Boykottversuchen durch die Arbeitgeber heute in praxi bei den meisten Gewerkschaften - wenn auch manchmal mit zeitlichen Verzögerungen - zu funktionieren. Damit steht der Mehrzahl der nigerianischen Gewerkschaften zum ersten Mal eine regelmäßige Einkommensquelle zur Verfügung. Dies ist angesichts der früheren Knappheit an finanziellen Mitteln, die den gewerkschaftlichen Handlungsspielraum stark einengte und zudem oft zur finanziellen Abhängigkeit vom Arbeitgeber und damit zur Korruption führte, eine wichtige Errungenschaft. De facto führt dieses System, da in gewerkschaftlich organisierten Betrieben meist niemand aus den Gewerkschaften austritt, aber of t auch zu nur formaler Mitgliedschaft. Gewerkschaftsmitglieder wissen mitunter gar nicht, daß sie organisiert sind. Die sich hieraus ergebenden Probleme anzupacken, wird eine wichtige Aufgabe für die Einzelgewerkschaften sein.

Die regelmäßigen Mitgliedsbeiträge erlaubten es vielen Gewerkschaften in den letzten Jahren die für eine erfolgreiche Arbeit notwendige Organisation aufzubauen. So besitzen die meisten von ihnen eine nationale Zentralverwaltung, der die meist in Regionen und Einzelbetriebe aufgeteilten gewerkschaftlichen Organisationseinheiten untergliedert sind. Um einen groben Eindruck von der Organisationsstruktur einer nigerianischen Gewerkschaft zu gewinnen, soll beispielhaft die der mittelgroßen Textilgewerkschaft vorgestellt werden (62 000 Mitglieder). In ihrer Zentralverwaltung sind 12 Hauptamtliche (inklusive Schreibkräfte) beschäftigt, weitere von der Gewerkschaft bezahlte Fachkräfte sind für die einzelnen Regionen angestellt. Alle anderen für die Gewerkschaft Tätigen arbeiten entweder ehrenamtlich oder werden von ihrem Betrieb bezahlt. In einem gewerkschaftlich gut organisierten Großbetrieb mit 4000 Lohnabhängigen gibt es etwa 9 Hauptamtliche, die bei voller Lohnzahlung von ihrer betrieblichen Tätigkeit freigestellt sind und 50 nebenamtlich Tätige. ${ }^{18}$ Der Großteil der Einnahmen der Textilgewerkschaft steht ihrer zentralen Verwaltung zur Verfügung (78 \%). ${ }^{19}$

Die einzelnen Regionen erhalten nur $2 \%$, die Betriebsgewerkschaften $10 \%$ und der NLC schließlich ebenfalls $10 \%$. Knapp die Hälfte der Mittel der Zentrale entfällt auf Personalausgaben, nur etwas mehr als $2 \%$ wird für gewerkschaftliche Fortbildung ausgegeben.

Die Verteilung der Ausgaben zwischen Zentralverwaltung und den regionalen/betriebli-

16 De iure gibt es also keine "closed shops",. d. h. die Pflicht eines Beschäftigten eines Betriebes in einer bestimmten Gewerkschaft organisiert zu sein. In der Praxis scheint aber von der Möglichkeit des Austritts aus einer Gewerkschaft nur selten Gebrauch gemacht zu werden.

17 Vgl. etwa die Verfassungen der nigerianischen Automobil- und Bauarbeitergewerkschaften, jeweils $\S 4$.

18 Eigene Erhebung im August/September 1980.

19 Dieser Prozentsatz liegt bei den anderen Gewerkschaften etwas niedriger, Angaben nach der Verfassung der Textilarbeitergewerkschaft, $\S 16$, IV. 
chen Gewerkschaften spiegelt den Wandel von der Hausgewerkschaft zur Industriegewerkschaft, der sich in den letzten Jahren vollzogen hat, deutlich wider. Den Gewerkschaften auf Betriebsebene bleibt nur wenig finanzieller Spielraum; immer wieder wird von ihnen, wohl zu Recht, beklagt, daß die Eigeninitiative der Gewerkschaftsbasis so unnötigerweise eingeschränkt wird.

Insgesamt stellt aber die solidere finanzielle Grundlage und die organisatorische Neustrukturierung für die Einzelgewerkschaften und den NLC eine gegenüber früher um vieles verbesserte Ausgangsbasis dar, gewerkschaftliche Forderungen geltend zu machen und insbesondere eine aktivere Tarifpolitik zu betreiben.

\section{Tarifpolitik}

Bis zur Neustrukturierung der Gewerkschaften waren Tarifauseinandersetzungen über 30 Jahre lang von einer nigerianischen Eigenart gekennzeichnet, den staatlich eingesetzten "Lohnkommissionen". Diese setzten im Abstand von zumeist 4-5 Jahren die Löhne für den staatlichen Sektor fest. ${ }^{20}$ Die Einsetzung dieser Kommissionen wurde von den Gewerkschaften im staatlichen Sektor durch Agitation, Streiks und Go-slows ${ }^{21}$ und oft auch durch die Ausübung politischen Drucks erreicht. Nach Festsetzung der Löhne im Staatssektor versuchten dann die vielen Hausgewerkschaften des Privatsektors durch eine Welle gewerkschaftlicher Kampfmaßnahmen, das dort erreichte Lohnniveau auf betrieblicher Ebene für ihre Mitglieder ebenfalls durchzusetzen. Ergebnis dieser Lohnkämpfe war dann oft eine sprunghafte Anhebung des Lohnniveaus (1974 z. B. um 30-120\%), das in den folgenden Jahren von der Inflation aber rasch wieder egalisiert wurde. ${ }^{22}$

Für die Gewerkschaften im staatlichen Sektor hatte dieses "Kommissionsmodell" den Vorteil, sich in der Regel nicht auf lange Tarifkämpfe einlassen zu müssen, zu denen sie weder finanziell noch organisatorisch gerüstet waren, sondern ihre Vorstellungen "nur" gegenüber den Kommissionen durchsetzen zu müssen. Die organisatorisch und finanziell noch schwächeren Gewerkschaften im privaten Sektor konnten sich an die im Staatssektor erreichten Abschlüsse dann oft "anhängen".

Trotz zeitweise beachtlicher Lohnschübe brachte dieses Lohnkommissionssystem der Masse der Lohnarbeiter in den letzten 15 Jahren keinen Reallohnzuwachs.

Jahreslohn eines ungelernten Arbeiters (in Naira) ${ }^{23}$

20 Vgl. im einzelnen Cohen, a.a.O.; Mitglieder bzw. Vorsitzende dieser Kommissionen waren of thöhere Bundesrichter.

21 Arbeit nach Vorschrift.

22 Vgl. nachfolgende Tabelle.

23 In Preisen von 1973; Angaben nach: Federal Republic of Nigeria, Federal Office of Statistics, Lagos, Annual Abstract of Statistics $1964 \mathrm{ff}$. 
Das starke Anwachsen der Löhne 1974 ist auf die Tätigkeit der ,Udoji-Lohnkommission zurückzuführen. In den ff. Jahren sinkt der Reallohn aber wieder stark ab. Da in den gleichen 15 Jahren sich praktisch der Aufstieg Nigerias zur Erdölgroßmacht vollzog, der allein im vergangenen Jahr ungefähr 25 Mrd. Dollar in die Kassen der Zentralregierung spülte (dies entspricht etwa $80 \%$ ihrer Gesamteinnahmen), ist dies zugleich ein Indiz dafür, daß eine gesamtgesellschaftliche Verteilung des nationalen Reichtums bis zum Ende der Militärregierung (1979) nicht stattgefunden hat.

Die neustrukturierten nigerianischen Gewerkschaften haben allerdings schon in den Jahren 1978/79 erste Versuche unternommen, die Lebensbedingungen der Lohnabhängigen zu verbessern und seit Amtsantritt der Zivilregierung wichtige Erfolge in den Tarifverhandlungen errungen. Die Bedeutung des erwähnten "Lohnkommissionssystem" bzw. das Ausmaß in dem die an einem Tarifkonflikt Beteiligten auf dessen Konfliktlösungsmechanismen zurückgreifen, scheint aber im Schwinden begriffen zu sein oder zumindest wichtigen Änderungen zu unterliegen.

Zwar richten die Gewerkschaften (bzw. genauer der NLC) nach wie vor zuerst ihre Forderungen etwa nach Heraufsetzung des Mindestlohns an die Regierung und nachdem diese Zugeständnisse gemacht hat (die prinzipiell auch für die Unternehmen des privaten Sektors gelten sollen), werden diese von den Industriegewerkschaften des privaten Sektors mehr oder weniger detailgetreu durch Einzeltarifverträge mit den jeweiligen Vertretern der Unternehmen einer Branche "festgeklopft".

Aber mindestens bisher sind keine Lohnkommissionen im eigentlichen Sinn wie in früheren Jahren, etwa unter der Leitung eines Richters, eingesetzt worden. Vielmehr haben Präsident Shagari und insbesondere die Nationalversammlung (d. h. das Repräsentantenhaus und der Senat) ihrer verfassungsgemäßen Kompetenz entsprechend, selbst versucht, Lösungsvorschläge gegenüber den Forderungen des NLC zu erarbeiten. ${ }^{24}$

Noch in der Zeit der Militärregierung hat der NLC im Mai 1979 zum ersten Male für ganz Nigeria einheitliche Forderungen aufgestellt, die er ultimativ an die Regierung

24 Vgl. aber die Stellungnahme der 9 Gouverneure (der Oppositionsparteien), die am 20. 5. 81 für die Einsetzung einer Lohnkommission plädierten; New Nigerian, 20. 5. 81. 
richtete. Gefordert wurde eine Beendigung des Lohnstopps bzw. staatlich reglementierter Lohnzuwächse, ${ }^{25}$ Aufhebung der restriktiven Arbeitsgesetzgebung, ${ }^{26}$ generelle Zahlung eines Miet- und Transportzuschusses für alle Arbeitnehmer und die sofortige Inkraftsetzung aller von den Einzelgewerkschaften schon ausgehandelten Tarifabkommen durch das Arbeitsministerium. Nach Verhandlungen des NLC mit der scheidenden Militärregierung wird die weitgehende Erfüllung' dieser Forderungen zugesagt, ohne daß konkrete Schritte in diese Richtung unternommen werden. Die im Oktober 1979 eingesetzte Zivilregierung unter Präsident Shagari zieht die Aufhebung der staatlichen Lohnreglementierung sogar wieder zurück, übernimmt aber ansonsten die alten Vereinbarungen. Als aber deren praktische Verwirklichung auch von der neuen Zivilregierung immer weiter hinausgeschoben wird, setzt ihr der NLC Ende Februar 1980 ein neues Ultimatum. Zugleich veröffentlicht er einen umfangreichen gewerkschaftlichen Forderungskatalog ("Workers' Charter of Demands"). Der konkrete tarifpolitische Forderungskatalog wird um die Forderung nach Erhöhung der Mindestlöhne auf 300 Naira/Monat erweitert. Da die Einzelgewerkschaften hinter dem NLC stehen und auch ein landesweiter Streik nicht ausgeschlossen erscheint, sieht sich die Regierung gezwungen einzulenken. Unter anderem erhöht sie die Mindestlöhne von 66 Naira auf 100 und gewährt für alle Staatsangestellten generelle Miet- und Transportzuschüsse von je 10 Naira/Monat.

Die auch gewerkschaftsintern nicht unstrittige Frage einer generellen Lohnerhöhung auf mindestens 300 Naira soll von einem Gremium aus Unternehmern, Regierung und Gewerkschaften erörtert werden. Regierung und Gewerkschaften fordern zudem die Unternehmer und Gewerkschaften des privaten Sektors auf, die schon erreichten Tarifabschlüsse im Staatssektor zu übernehmen. Wichtige Einzelgewerkschaften (so die Bau-, Textil-, Nahrungsmittelgewerkschaften) können in den ff. Monaten auch vergleichbare Tarifabschlüsse durchsetzen. Durch Kurzstreiks und v. a. Go-slows können sie zudem zahlreiche soziale Verbesserungen erreichen, wie Urlaubsbestimmungen (14-18 Tage), Mutterschutzregelungen und Lohnfortzahlung im Krankheitsfalle etc. Die Bedeutung dieser Tarifabkommen liegt auch darin, daß viele von ihnen schriftlich fixiert und zum ersten Mal landeseinheitlich für einen bestimmten Industriezweig abgeschlossen werden. Gegenüber diesen Einzelerfolgen, kommt die Arbeit der Kommission, die über die 300Naira-Minimallohnforderung beraten soll, nicht voran. Nach einem erneuten Ultimatum des NLC übernimmt es endlich der Arbeitsausschuß des nigerianischen Parlaments, weitere Vorschläge für die Anhebung der Mindestlöhne zu erarbeiten.

Nach immer neuen Verzögerungen beschließt der NLC ein halbes Jahr später schließlich einen landesweiten Streik zur Durchsetzung seiner Forderungen auszurufen. Präsident Sunmonu glaubt den NLC für diesen dritten Generalstreik in der nigerianischen Geschichte $^{27}$ umso mehr gerüstet, als er und seine Führungsmannschaft auf dem Gewerk-

25 Insbesondere diese Maßnahmen haben das starke Absinken der Reallöhne verursacht. Die Militärregierung hatte 1977/78 nur Lohnerhöhungen von $7 \%$ zugelassen und 1978/79 einen generellen Lohnstop verhängt: 1979/80 waren $10 \%$ Lohnerhöhung vorgesehen.

26 Im einzelnen vgl. Ausführungen weiter unten.

27 Die beiden anderen fanden 1945 und 1964 statt. 
schaftstag im Februar 1981 in Kano mit großer Mehrheit gegen seinen innergewerkschaftlichen Gegner Ojeli von der Nigerian Civil Service Union wiedergewählt wurde. ${ }^{28}$ Tatsächlich ist der nur 2 Tage dauernde Ausstand nur teilweise erfolgreich. Zwar kommt fast der gesamte öffentliche Verkehr zum Erliegen, die meisten Gewerkschaften - und hier insbesondere die im Süden des Landes - beteiligen sich; aber einige wichtige Gewerkschaften wie die Nigerian Civil Service Union oder die Gewerkschaft der Olarbeiter nehmen nicht teil. Im Norden des Landes ist außerdem die Beteiligung geringer. ${ }^{29}$ Gegenüber der zumindest stillschweigenden Unterstützung einzelner Oppositionsparteien (UPN) hat der NLC mit der Gegnerschaft der weitaus meisten Zeitungen und der Regierungsparteien zu rechnen. ${ }^{30}$

Unmittelbares Ergebnis des Streiks ist somit nur die Heraufsetzung der Mindestrente von 33 auf 50 Naira pro Monat und die Wiederherstellung von Automobildarlehen und -zuschüssen für Staatsangestellte. Die für die Gewerkschaften entscheidende Frage der Heraufsetzung der Mindestlöhne wird wieder (nur) an den Senat und das Repräsentantenhaus verwiesen. ${ }^{31}$

NLC und Einzelgewerkschaften haben so Mitte 1981 - nicht zuletzt auf Grund ihrer organisatorisch und finanziellen Neustrukturierung - zwar die Einlösung einiger wichtiger Forderungen erreicht, eine endgültige Einigung über die Höhe des Mindestlohns steht aber noch aus. Eine aktive Gewerkschaftspolitik muß sich aber in Zukunft verstärkt mit zwei Problemkreisen auseinandersetzen, deren Implikationen im folgenden aufgezeigt werden sollen: der Rolle des Staates im Sozial- und Arbeitsrecht und der unzureichenden gewerkschaftlichen Bildungsarbeit.

\section{Staatliche Interventionen im Sozialrecht und Arbeitsrecht}

Ein staatliches, soziales Sicherungsnetz für Arbeitnehmer fehlt in Nigeria oder ist ineffizient.

So gibt es zwar schon seit 1957 ein Gesetz zum Schutz von Gesundheit und Sicherheit der Arbeitnehmer in den Betrieben (Workmens' Compensation Act); in praxi wird es aber kaum angewandț. Ebenfalls seit längerer Zeit besteht eine gesetzliche Altersversorgung (National Provident Fund), zu der Arbeitnehmer und Unternehmer jeweils $50 \%$ beitragen; ihre Funktion ist aber durch statutorische und organisatorische Mängel stark beeinträchtigt. Krankenversicherung, Arbeitslosenunterstützung und andere Sozialversicherungssysteme fehlen völlig. Betriebliche Sicherungssysteme haben diesen Mangel nur sehr unzureichend und nicht für die vielen Lohnabhängigen der Klein- und Kleinstbetriebe beheben können.

28 Daily Times, 28. 2. 81.

29 Im einzelnen vgl. etwa: Nigeria Newaletter vom 25. 5. 81; West Africa vom 18. 5. 81; Punch vom 14. 5. 81 und New Nigerian vom 14. 5. 81 .

30 Sh. unter "Gewerkschaften als pol. Einflußfaktor".

31 Vgl. die Vorschläge des Senats auf Heraufsetzung des gesetzlichen Mindestlohns auf 125, - Naira und die demgegenüber (feste) Haltung des Repräsentantenhauses, Daily Times und National Concord vom 16. 6. 81. 
Im arbeitsrechtlichen Bereich bestehen dagegen aus der Zeit der Militärregierung viele, die Gewerkschaftsarbeit stark behindernde, Gesetze, die trotz zahlreicher Zusagen der Zivilregierung immer noch nicht aufgehoben sind. Einige dieser Gesetze, wie das "Trade Union Decree" von 1973 verbieten gewerkschaftliche Organisierung in "sensitiven" Bereichen und stehen damit in Widerspruch zu Ártikel 37 der neuen Verfassung, die Organisationsfreiheit gewährleistet.

Zentralstück des Arbeitsrechts ist aber ein durch Decree No 7/76 und 54/77 verankertes Zwangsschlichtungsverfahren. Darin wird jede Arbeitskampfmaßnahme für illegal erklärt, wenn nicht zuvor zur Beilegung des Konflikts zahlreiche vom Arbeitsministerium bestimmte Schlichtungsinstanzen und abschließend das höchste Arbeitsgericht angerufen worden sind. So wurden Tarifforderungen des Krankenhauspersonals vom Arbeitsministerium z. B. 1 Jahr lang bis zum August 1980 verschleppt, bevor das Arbeitsgericht den Streitfall zugewiesen bekam. Jedes Tarifergebnis muß zudem vom Arbeitsministerium bestätigt werden. Durch diese und weitere gesetzliche Möglichkeiten kann somit der Staat legal jede gewerkschaftliche Aktivität stark einschränken. Da er gleichzeitig der größte Arbeitgeber ist, hat er praktisch eine Doppelrolle inne, die Interessenkonflikte unvermeidlich machen.

\section{Gewerkschaftliche Bildungsarbeit}

Ein Problemkind besonderer Art ist die gewerkschaftliche Bildungsarbeit, obgleich die Einzelgewerkschaften wie auch der NLC in letzter Zeit verstärkt versucht haben, das Angebot an Schulungskursen für ihre Mitglieder und Funktionäre zu vergrößern. Das Problem ist vielschichtig. Viele Lohnabhängige sind an die Anforderungen des industriellen Arbeitsprozesses nicht gewöhnt. Die meisten, die etwa in den großen Textilfabriken im Norden arbeiten, kommen mehr oder weniger direkt aus ländlichen Gebieten. Bis zu $50 \%$ von ihnen verlassen innerhalb eines Jahres in Folge von Eingewöhnungsschwierigkeiten ihren Arbeitsplatz wieder. ${ }^{32}$

Gewerkschaftliche Organisierung bzw. aktive Mitarbeit in den Gewerkschaften sind neu für sie. In der momentan noch herrschenden Atmosphäre des wirtschaftlichen Booms in Nigeria scheint für manche von ihnen der individuelle Aufstieg - Eröffnung eines Kleingewerbes - erstrebenswerter als Mitarbeit in den Gewerkschaften.

Bei einer hohen Analphabetenrate können zudem auch von den Gewerkschaften herausgegebene Zeitungen/Zeitschriften wie dies bei den Gewerkschaften im Gaststätten- und Textilgewerbe geschieht, nur einen Teil der Mitglieder erreichen.

Die Schulung von Mitgliedern findet - soweit vorhanden - bei den Einzelgewerkschaften daher eher auf Versammlungen statt. Fortbildung von neben/hauptamtlichen Gewerkschaften wird dagegen in mehrtägigen Trainingskursen, die manchmal in Verbindung mit lokalen Universitäten abgehalten werden, betrieben. Da die Mittel für den Bildungs- 
etat äußerst begrenzt sind, sind die Einzelgewerkschaften dabei oft auf die finanzielle Unterstützung der Arbeitgeber angewiesen.

Der NLC veranstaltet ebenfalls im Rahmen seiner begrenzten finanziellen Möglichkeiten Trainingskurse, zum Teil in Zusammenarbeit mit den Universitäten von Lagos und Ibadan. Darüber hinaus vermittelt er Auslandsstipendien (1979 z. B. insgesamt 70), die von östlichen und westlichen Institutionen vergeben werden..$^{33}$ Die Nachfrage nach diesen Schulungsmöglichkeiten bei nigerianischen Gewerkschaften übersteigt aber bei weitem das Angebot. Die (Wieder)Eröffnung eines gewerkschaftseigenen Bildungsinstituts (National Institute for Labour Studies) in Illorin ist zwar von der Zentralregierung seit Jahren zugesagt worden und im Juni 1980 wurden sogar 1/2 Mio Naira bereitgestellt; der praktischen Verwirklichung ist dieses Projekt aber nur wenig näher gerückt.

Neben unzulänglichen Mitteln, die die Gewerkschaften immer wieder auf die Unterstützung der Arbeitgeber/Regierung verweisen, fehlt es oft auch an geeigneten Unterrichtsmaterialien, da die vorhandenen zumeist veraltet sind und von (europäischen) Modellvorstellungen ausgehend zuwenig die nigerianische Realität berücksichtigen.

Bevor versucht werden soll in einer abschließenden Einschätzung die Perspektiven gewerkschaftlicher Arbeit in Nigeria zu bestimmen, muß ihre Stellung im politischen System Nigerias noch verortet werden.

\section{Gewerkschaften als politischer Einflußfaktor}

Generell kann gesagt werden, daß sich die Gewerkschaften in den letzten Jahren nach den Parteien und neben den zurückgetretenen Militärs zum wichtigsten gesellschaftlichen Machtfaktor entwickelt haben. Die Gewerkschaften stellen aber keinen einheitlichen Block dar. Noch heute bestehen alte Spannungen, die früher in der Spaltung der Gewerkschaftsbünde in den ULC, der Mitglied des IBFG war, und in den NTUC, der Mitglied im WGB war, ihren Ausdruck fanden.

In jüngster Zeit mehren sich auch Versuche von interessierter Seite, ${ }^{34}$ politische Spannungen zwischen den Einzelgewerkschaften und innerhalb des NLC zu vertiefen.

Eine ähnliche enge Verflechtung der Gewerkschaften mit einer Partei, wie sie in vielen europäischen Industrieländern existiert, gibt es in Nigeria nicht. Die fünf 1979 entstandenen Parteien Nigerias werden - trotz einiger verfassungsrechtlicher Versuche, dies zu ändern - immer noch bestimmten Stammesgruppen bzw. Regionen zugerechnet.

Bei der Befragung von Gewerkschaftern bezüglich ihrer Parteipräferenz, fällt eine deutliche Zurückhaltung auf. ${ }^{35}$ Noch am häufigsten wird die UPN und PRP genannt, die ih-

33 Angabe von Dr. E. Taiwo, Head Education \& Training Dept. NLC, 23. 9. 80.

34 Vergleiche die oft tendenziöse Berichterstattung im New Nigerian oder die Glossen von Umoh James Umoh in der Daily Times (größte Zeitung des Landes, regierungsnahestehend), der z. B. die auf dem Gewerkschaftstag im Februar 1981 erfolgte Wiederwahl Sunmonus als Kampf zwischen "Demokratie und Marxismus" zu verhindern suchte. Demgegenüber gibt es nur wenig Medien, die den Gewerkschaften "freundlich" gegenüber stehen; meist stehen sie wie der Nigerian Tribune oder die Sketch der UPN nahe.

Eigene Erhebungen von August-Oktober 1980. 
ren Einflußbereich im Westen und Norden haben. Zum Teil wird auch die Regierungspartei NPN genannt, die sich am ehesten auf ein überregionales Wählerrevoir stützen kann; weniger häufig dagegen die NPP und die GNPP.

Diese relativ breite Streuung der Parteienmitgliedschaft bzw. Präferenz von Gewerkschaftsmitgliedern spiegelt ein Dilemma der Gewerkschaften wider. Zum einen ist damit u. U. die Durchsetzung gewerkschaftspolitischer Forderungen - wie etwa der nach Aufhebung der restriktiven Arbeitsgesetzgebung aus der Militärzeit - erschwert.

Zum anderen würde aber jeder Versuch einer stärkeren Anlehnung der Gewerkschaften an nur eine Partei zu innergewerkschaftlichen Spannungen führen, weil die Zugehörigkeit zu einer Partei immer noch mit der Zugehörigkeit zu einer bestimmten Volks- oder Stammesgruppe gleichgesetzt wird. So war im August 1980 ein Streik des Krankenhauspersonals dem Vorwurf aufgesetzt, die Gewerkschaft verfolge damit das Ziel die UPN zu unterstützen.

Ein ähnlicher Vorwurf wurde auch während des Generalstreiks im Mai 1981 erhoben. ${ }^{36}$ Eine Abspaltung einzelner Industriegewerkschaften, die auf Grund dieser politischen Spannungen vorausgesagt wurde, kann daher nicht ausgeschlossen werden. ${ }^{37}$

\section{Entwicklungsperspektiven der nigerianischen Gewerkschaften}

Praktische Konsequenz dieser Schwierigkeiten ist, daß sich viele Gewerkschaftsmitglieder bei den Wahlen 1979 auf verschiedenen Parteilisten in die parlamentarischen Gremien und insbesondere in den wichtigen Senatsausschuß für Arbeit wählen ließen, ihre Mitgliedschaft bei den jeweiligen Einzelgewerkschaften für die Dauer ihrer aktiven Parteitätigkeit aber ruht.

Vorrang in der Aufgabenstellung der nigerianischen Gewerkschaften wird weiterhin die Anhebung des noch geringen Lohnniveaus und der Aufbau eines sozialen Sicherungsnetzes für die Arbeitnehmer haben. Dies gilt insbesondere für die große Menge der Taglöhner ("daily-rated-workers") und der Arbeitnehmer in bisher wenig gewerkschaftlich organisierten Kleinbetrieben, deren Lebens- und Arbeitsbedingungen besonders schlecht sind.

Wichtige Grundsteine für eine erfolgreiche gewerkschaftliche Arbeit und auch die Erfüllung weitergehender Forderungen sind mit der organisatorisch/finanziellen Neustrukturierung gelegt.

Nach wie vor ist aber die gerade erst errungene Einheit der gewerkschaftlichen Organisation auf Grund ethnischer und politischer Spannungen innerhalb des NLC und der Einzelgewerkschaften gefährdet. Einige Anzeichen deuten darauf hin, daß diese Tenden-

36 Daily Times, 14. 5. 81.

37 Vergleiche die nach dem Gewerkschaftstag von Kano im Februar 1981 erfolgte Gründung eines "Committee for Democratic Trade Unionism" und die von Umoh (West Africa, 19. 1. 81) für den Fall der Wiederwahl Sunmonus vorausgesagte Abspaltung wichtiger Industriegewerkschaften, wie der Nigerian Civil Service Union. 
zen auch von staatlichen Stellen gegenüber den mächtiger werdenden Gewerkschaften gefördert werden.

Demgegenüber geht es den nigerianischen Gewerkschaften um eine Einschränkung der weitreichenden Eingriffskompetenzen des Staates in rein gewerkschaftliche bzw. tarifrechtliche Bereiche.

Die Gewinnung des hiefür nötigen Handlungsspielraums wird auch abhängig davon sein, wieweit es den Gewerkschaften gelingt, Mitglieder und Basis zu aktivieren und motivieren.

Die in den letzten Jahren rasch zunehmende Mitgliederzahl und der Ausbau der gewerkschaftlichen Bildungsarbeit, der noch forciert werden muß, könnten dabei wichtige Ausgangsbedingungen sein.

Innergewerkschaftlich wird es auch wichtig sein, der durch die Umstellung von Hausgewerkschaften auf Industriegewerkschaften möglichen Entstehung und Verkrustung von Hierarchien entgegenzuwirken und ein Ausbreiten der in Nigeria allgegenwärtigen Korruption in den Gewerkschaften zu verhindern. ${ }^{38}$

\section{Abkürzungsverzeichnis}

NLC Nigerian Labour Congress; entspricht in seiner Funktion etwa dem DGB

ULC United Labour Congres und Nigerian Trade Union

NTUC Council; die beiden größten gew. Dachverbände in Nigeria vor der Neustrukturierung

WGB Weltgewerkschaftsbund

IBFG Internationaler Bund Freier Gewerkschaften

UPN United Party of Nigeria

PRP People's Redemption Party

NPN National party of Nigeria

NPP Nigerian People's Party

GNPP Great Nigerian People's Party

38 Vgl. für die Zeit der Militärregierung insb.: Adebiyi-Report, Lagos 1977; Guy Arnold: Modern Nigeria, London, 1977, S. 144 f.; in jüngster Zeit sind Korruptionsvorwürfe v. a. in Bezug auf den Gewerkschaftstag im Februar 1981 laut geworden auf dem sich Sunmonu gegen Ojeli durchsetzen konnte. Für die wechselseitigen Vorwürfe sh. etwa: Nigerian Tribune vom 14. 5. 81 und West Africa vom 27.4.81. 
The Six-Year Plan does not change this policy fundamentally. Although the industrial policy has to create more employment opportunities than in earlier plans and defines the necessity to increase the efficiency of human capital, the educational policy sticks to traditional guidelines and historical patterns. It has to be concluded that the radical change in the educational system - required by the industrialization policy - has not been realized yet. Insofar adjustments, proposed f.i. by the expert mission of the ILO, are not yet part of the Sudanese policy.

The example of the Sudan is representative for the industry and education policy in many least developed countries. One reason for neglecting the interrelationship between labour market and training is the lack of statistical analysis of demand on supply of manpower and the low efficiency in planning, performance and evaluation of educational policy according to the demand of the industry in the development process. Developed countries can support especially least developed countries to overcome these difficulties which hamper self-sustaining growth by providing personal, know-how and financial resources for manpower analysis and planning and educational policy.

\section{Trade Unions in Nigeria since 1977 . . . By Christoph Muermann}

Nigerian Trade Unions have developed in recent years to one of the most powerful Trade Union organizations in Africa.

What are their limitations and perspectives? The ans wer to this question might be interesting not only for Nigerian Trade Unionists - especially in a time of internationalization of production.

The rapid industrial development in Nigeria, which is based on the "oil-boom", has promoted on the one hand the growth of a capital-intensive industry which hardly takes into accout basic needs. On the other hand it has also promulgated social problems such as the influx of the rural population and even immigration workers from neighbouring countries into the industrial areas who are trying to participate in the economic growth, but often only augment mass unemployment in the urban slum areas. The still existing split of the Nigerian economy into a modern sector and a traditional sector (with different wages) and the short historical consciousness of a relatively young Trade Union Movement are among the other factors, which determine the limitations of Nigerian Trade Unions.

Nevertheless the establishment of one Central Labour Organization (Nigerian Labour Congress) in Nigeria during the end of the military government in 1977/78 with 42 member Trade Unions (organised on an industrial basis) and the establishment of a more solid financial contribution system means a big step forwards for the Nigerian Trade Union Movement. 
The significance of this development for the local and central Trade Union level and especially the legal structure behind this system is shown in this article as well as its effects on the wage- determination process and the recent interferences of the political system.

Finally two basic problems are decribed, which Nigerian Trade Unions will have to tackle in the future: The development of a functioning social insurance system and the need for a more effective educational system for Trade Unionists.

The article is based on findings made during a stay of some months in Nigeria during the latter part of 1980; data up to the middle of 1981 are taken into account. 